\title{
RARE EVENTS, TEMPORAL DEPENDENCE, AND THE EXTREMAL INDEX
}

\author{
JOHAN SEGERS, ${ }^{*}$ Tilburg University
}

\begin{abstract}
Classical extreme value theory for stationary sequences of random variables can to a large extent be paraphrased as the study of exceedances over a high threshold. A special role within the description of the temporal dependence between such exceedances is played by the extremal index. Parts of this theory can be generalized not only to random variables on an arbitrary state space hitting certain failure sets, but even to a triangular array of rare events on an abstract probability space. In the case of M4 (maxima of multivariate moving maxima) processes, the arguments take a simple and direct form.
\end{abstract}

Keywords: Block maximum; exceedance; extremal index; failure set; mixing condition; M4 process; rare event; stationary sequence

2000 Mathematics Subject Classification: Primary 60G70

Secondary $62 \mathrm{G} 32$

\section{Introduction}

Many applied sciences require the handling of events with low probability but large, often disastrous impact. Of particular interest is the way in which such rare events interact: an unusually stormy day at a particular site might be followed by another one at the same or a neighboring site, while a large drop in a stock index might trigger similar negative movements in the next time period for the same or other financial time series. What are the principles governing these dependencies?

The theory developed in this paper is inspired by a concept from classical extreme value theory. A stationary sequence of random variables, $\left\{X_{n}\right\}$, is said to have extremal index $\theta \in[0,1]$ if, for every $\tau, 0<\tau<\infty$, there exists a sequence of thresholds, $\left\{u_{n}\right\}$, such that $n \operatorname{Pr}\left(X_{1}>u_{n}\right) \rightarrow \tau$ and $\operatorname{Pr}\left(\max _{i=1, \ldots, n} X_{i} \leq u_{n}\right) \rightarrow \exp (-\tau \theta)$ as $n \rightarrow \infty$ (Leadbetter (1983)). The extremal index $\theta$ quantifies the strength of dependence between threshold exceedances $\left\{X_{i}>u_{n}\right\}$, with $\theta=1$ corresponding to asymptotic independence and $\theta \downarrow 0$ to an increasing propensity of large observations to occur in clusters. In the context of multivariate time series, the extremal index makes its appearance in the asymptotic distribution of the vector of componentwise maxima (Nandagopalan (1994), Smith and Weissman (1996), Perfekt (1997), Beirlant et al. (2004, Chapter 10), Zhang and Smith (2004)).

As already hinted at in Nandagopalan (1994), we can in fact start from a stationary process on an arbitrary state space in which a sequence of failure sets represents ever more extreme states for the process. The extremal index, which now also depends on the sequence of failure sets, describes the strength of temporal dependence between failure set hits. An even further abstraction is possible, to a triangular array of events every row of which satisfies a certain stationarity condition.

Received 31 March 2003; revision received 30 January 2006.

* Postal address: Department of Econometrics and Operations Research, Tilburg University, PO Box 90153, NL-5000

LE Tilburg, The Netherlands. Email address: jsegers@uvt.nl 
For a single row of events, the following quantities are of interest: the probability that none of the events occurs; the probability that the occurrence of an event is not followed in the near future by another one; the mean number of events that occur given that there occurs at least one; and, conditionally on the occurrence of an event, the time until the occurrence of the next one. The relations between these quantities can be described in terms of various inequalities. These complement the assessment of the accuracy of the compound Poisson approximation for the empirical point process of exceedances in Barbour et al. (2002). Furthermore, these inequalities lead to asymptotic results that serve on the one hand to formulate, in the framework of rare events, known characterizations of the extremal index (Leadbetter (1983), O'Brien (1987), Ferro and Segers (2003)), and on the other hand to complement various Poisson limit results for triangular arrays (Hüsler (1993), Hüsler and Schmidt (1996)). Point process results will not be pursued in this paper, as the dependence restrictions in force will be weaker than those in the aforementioned papers.

The exposition starts in Section 2 with a discussion of the multivariate extremal index of M4 (maxima of multivariate moving maxima) processes. In this relatively simple example, short and direct arguments suffice to illustrate the more general theory. By way of an intermediate step, results for a stationary sequence in an arbitrary state space are formulated, in Section 3. The highest level of abstraction is reached in Sections 4-6. The set-up and the notation used are detailed in Section 4. The core of the paper is Section 5, containing asymptotic theory for dependence within a triangular array of rare events. The theory is based on a meticulous analysis leading to sharp inequalities, in Section 6. Finally, the appendices contain some technical arguments. Where not specified explicitly, all limits hold as $n \rightarrow \infty$ in the set of positive integers.

\section{Maxima of multivariate moving maxima}

M4 processes provide an instructive example of how phenomena in the context of extremes of univariate stationary processes carry over to a more general setting. For such processes, direct arguments suffice to reveal the connection between the extremal index and temporal dependence between exceedances over high multivariate thresholds.

\subsection{M4 processes}

A $d$-variate random sequence $X_{i}=\left(X_{i, 1}, \ldots, X_{i, d}\right)$, where $i \in \mathbb{Z}$ (the set of integers), is called an $M 4$ process if it admits the representation

$$
X_{i, j}=\max _{l \geq 1} \max _{p \in \mathbb{Z}} a_{l, i-p, j} Z_{l, p} \quad \text { for } i \in \mathbb{Z} \text { and } j=1, \ldots, d ;
$$

the variables $Z_{l, p}$, where $l=1,2, \ldots$ and $p \in \mathbb{Z}$, are independent standard Fréchet random variables, that is,

$$
\operatorname{Pr}\left(Z_{l, p} \leq x\right)=\exp \{-1 / x\}, \quad 0<x<\infty,
$$

while the $a_{l, k, j}$ are nonnegative numbers such that

$$
\sum_{l \geq 1} \sum_{k \in \mathbb{Z}} a_{l, k, j}=1 \quad \text { for } j=1, \ldots, d .
$$

Note that the process $\left\{X_{i}: i \in \mathbb{Z}\right\}$ is constructed as the maximum of a sequence of multivariate moving maximum processes, whence the name 'M4'. 
The M4 process (2.1) is strictly stationary, its marginal distributions being standard Fréchet. The distribution function, $G_{m}$, of the $m d$-variate vector $\left(X_{1}, \ldots, X_{m}\right)$ is given by

$$
\begin{aligned}
G_{m}\left(x_{1}, \ldots, x_{m}\right) & =\exp \left\{-V_{m}\left(x_{1}, \ldots, x_{m}\right)\right\}, \quad \text { with } \\
V_{m}\left(x_{1}, \ldots, x_{m}\right) & =\sum_{l \geq 1} \sum_{p \in \mathbb{Z}} \max _{i=1, \ldots, m} \max _{j=1, \ldots, d} \frac{a_{l, i-p, j}}{x_{i, j}},
\end{aligned}
$$

for $x_{1}, \ldots, x_{m} \in(0, \infty]^{d}$. In particular, all finite-dimensional distributions of the process are simple max-stable, that is, $\left\{G_{m}\left(t x_{1}, \ldots, t x_{m}\right)\right\}^{t}=G_{m}\left(x_{1}, \ldots, x_{m}\right)$ for every $t, 0<t<\infty$. Such a process is called max-stable in the terminology of de Haan (1984).

M4 processes were introduced in Smith and Weissman (1996) to provide examples for the multivariate extremal index, to be defined below. See Zhang (2002) for applications of M4 processes to the modelling of financial time series.

\subsection{Temporal dependence between high-threshold exceedances}

An observation $X_{i}$ is said to exceed the threshold $x$ if $X_{i} \not \leq x$, that is, if $X_{i, j}>x_{j}$ for some $j=1, \ldots, d$. For M4 processes, we will analyse the temporal dependence between exceedances over threshold sequences of the form $n x$ that have $x_{j}>0$ for every $j=1, \ldots, d$.

For positive integers $n$ and for $x \in(0, \infty]^{d}$, let

$$
V_{n}(x):=V_{n}(x, \ldots, x)=\sum_{l \geq 1} \sum_{p \in \mathbb{Z}} \max _{i=1, \ldots, n} \max _{j=1, \ldots, d} \frac{a_{l, i-p, j}}{x_{j}} ;
$$

also let $V_{0} \equiv 0$. The following lemma is of great use in the study of the temporal dependence between extremes of an M4 process.

Lemma 2.1. For $x \in(0, \infty]^{d}$, the functions $V_{n}$ in (2.2) satisfy

$$
\lim _{n \rightarrow \infty}\left\{V_{n}(x)-V_{n-1}(x)\right\}=\lim _{n \rightarrow \infty} V_{n}(n x)=\sum_{l \geq 1} \max _{k \in \mathbb{Z}} \max _{j=1, \ldots, d} \frac{a_{l, k, j}}{x_{j}}=: W(x) .
$$

Proof. For $l \geq 1$ and $k \in \mathbb{Z}$, let $b_{l, k}=\max _{j=1, \ldots, d} a_{l, k, j} / x_{j}$. We have

$$
V_{n}(x)-V_{n-1}(x)=\sum_{l \geq 1} \sum_{p \in \mathbb{Z}}\left(\max _{i=1, \ldots, n} b_{l, i-p}-\max _{i=1, \ldots, n-1} b_{l, i-p}\right) .
$$

Writing $\lambda_{+}=\max (\lambda, 0)$ for $\lambda \in \mathbb{R}$, we obtain

$$
\begin{aligned}
V_{n}(x)-V_{n-1}(x) & =\sum_{l \geq 1} \sum_{p \in \mathbb{Z}}\left(b_{l, n-p}-\max _{i=1, \ldots, n-1} b_{l, i-p}\right)_{+} \\
& =\sum_{l \geq 1} \sum_{k \in \mathbb{Z}}\left(b_{l, k}-\max _{i=1, \ldots, n-1} b_{l, i+k-n}\right)_{+} \\
& =\sum_{l \geq 1} \sum_{k \in \mathbb{Z}}\left(b_{l, k}-\max _{i=1, \ldots, n-1} b_{l, k-i}\right)_{+} .
\end{aligned}
$$

By the dominated convergence theorem,

$$
\lim _{n \rightarrow \infty}\left\{V_{n}(x)-V_{n-1}(x)\right\}=\sum_{l \geq 1} \sum_{k \in \mathbb{Z}}\left(b_{l, k}-\max _{r<k} b_{l, r}\right)_{+} .
$$


The identity

$$
\sum_{k \in \mathbb{Z}}\left(b_{l, k}-\max _{r<k} b_{l, r}\right)_{+}=\max _{k \in \mathbb{Z}} b_{l, k}
$$

yields $\lim _{n \rightarrow \infty}\left\{V_{n}(x)-V_{n-1}(x)\right\}=W(x)$. Furthermore,

$$
V_{n}(n x)=\frac{1}{n} V_{n}(x)=\frac{1}{n} \sum_{k=1}^{n}\left\{V_{k}(x)-V_{k-1}(x)\right\} .
$$

Since the Cesàro transform of a converging sequence converges to the same limit as the original sequence, we also have $\lim V_{n}(n x)=W(x)$. This concludes the proof of Lemma 2.1.

For $x \in(0, \infty]^{d} \backslash\{(\infty, \ldots, \infty)\}$, let

$$
\theta(x)=\frac{W(x)}{V_{1}(x)}=\frac{\sum_{l \geq 1} \max _{k \in \mathbb{Z}} \max _{j=1, \ldots, d} a_{l, k, j} / x_{j}}{\sum_{l \geq 1} \sum_{k \in \mathbb{Z}} \max _{j=1, \ldots, d} a_{l, k, j} / x_{j}} .
$$

This $\theta$ is called the (multivariate) extremal index (function) of the M4 process (2.1). It inherits all the familiar properties of the extremal index of a univariate stationary process.

Theorem 2.1. Let $\left\{X_{n}\right\}$ be the M4 process (2.1). For $x \in(0, \infty]^{d} \backslash\{(\infty, \ldots, \infty)\}$,

$$
\begin{aligned}
\operatorname{Pr}\left(X_{i} \leq n x \forall i=1, \ldots, n\right) & =\left\{\operatorname{Pr}\left(X_{1} \leq n x\right)\right\}^{n \theta(x)}+o(1) \\
& \rightarrow \exp \{-W(x)\} .
\end{aligned}
$$

If $m_{n}$ is a positive integer sequence such that $m_{n} \rightarrow \infty$ and $m_{n}=o(n)$, then

$$
\mathrm{E}\left[\sum_{i=1}^{m_{n}} \mathbf{1}\left(X_{i} \not \leq n x\right) \mid \exists i=1, \ldots, m_{n}: X_{i} \not \leq n x\right] \rightarrow \frac{1}{\theta(x)} .
$$

If $s_{n}$ is a positive integer sequence such that $s_{n} \rightarrow \infty$ and $s_{n} / n \rightarrow \lambda \in[0, \infty]$, then

$$
\operatorname{Pr}\left(X_{i} \leq n x \forall i=2, \ldots, s_{n} \mid X_{1} \not \leq n x\right) \rightarrow \theta(x) \exp \left\{-\lambda V_{1}(x) \theta(x)\right\} .
$$

Proof. The proof relies on Lemma 2.1. First,

$$
\operatorname{Pr}\left(X_{i} \leq n x \forall i=1, \ldots, n\right)=\exp \left\{-V_{n}(n x)\right\} \rightarrow \exp \{-W(x)\}
$$

and

$$
\left\{\operatorname{Pr}\left(X_{1} \leq n x\right)\right\}^{n}=\exp \left\{-n V_{1}(n x)\right\}=\exp \left\{-V_{1}(x)\right\}
$$

Second,

$$
\begin{aligned}
\mathrm{E}\left[\sum_{i=1}^{m_{n}}\right. & \left.\mathbf{1}\left(X_{i} \not \leq n x\right) \mid \exists i=1, \ldots, m_{n}: X_{i} \leq n x\right] \\
& =\frac{m_{n} \operatorname{Pr}\left(X_{1} \not \leq n x\right)}{\operatorname{Pr}\left(\exists i=1, \ldots, m_{n}: X_{i} \not \leq n x\right)} \\
& =\frac{m_{n}\left[1-\exp \left\{-V_{1}(n x)\right\}\right]}{1-\exp \left\{-V_{m_{n}}(n x)\right\}} \\
& =\frac{n\left[1-\exp \left\{-(1 / n) V_{1}(x)\right\}\right]}{\left(n / m_{n}\right)\left[1-\exp \left\{-\left(m_{n} / n\right) V_{m_{n}}\left(m_{n} x\right)\right\}\right]} \\
& \rightarrow \frac{V_{1}(x)}{W(x)}
\end{aligned}
$$


Finally,

$$
\begin{aligned}
\operatorname{Pr}\left(X_{i}\right. & \left.\leq n x \forall i=2, \ldots, s_{n} \mid X_{1} \nless n x\right) \\
& =\frac{\operatorname{Pr}\left(X_{i} \leq n x \forall i=2, \ldots, s_{n}\right)-\operatorname{Pr}\left(X_{i} \leq n x \forall i=1, \ldots, s_{n}\right)}{1-\operatorname{Pr}\left(X_{1} \leq n x\right)} \\
& =\frac{\exp \left\{-V_{s_{n}-1}(n x)\right\}-\exp \left\{-V_{s_{n}}(n x)\right\}}{1-\exp \left\{-V_{1}(n x)\right\}} \\
& =\exp \left\{-V_{s_{n}}(n x)\right\} \frac{n\left[\exp \left\{V_{s_{n}}(n x)-V_{s_{n}-1}(n x)\right\}-1\right]}{n\left[1-\exp \left\{-V_{1}(n x)\right\}\right]} \\
& =\exp \left\{-\frac{s_{n}}{n} V_{s_{n}}\left(s_{n} x\right)\right\} \frac{n\left[\exp \left\{(1 / n)\left(V_{s_{n}}(x)-V_{s_{n}-1}(x)\right)\right\}-1\right]}{n\left[1-\exp \left\{-(1 / n) V_{1}(x)\right\}\right]} \\
& \rightarrow \exp \{-\lambda W(x)\} \frac{W(x)}{V_{1}(x)} .
\end{aligned}
$$

This concludes the proof of Theorem 2.1.

Equation (2.3), due to Smith and Weissman (1996), states that the role played by the extremal index in the asymptotic distribution of the componentwise sample maximum is exactly the same as in the original definition for univariate sequences in Leadbetter (1983). Take $x$ such that all coordinates but its $j$ th are equal to infinity to arrive at the result that the extremal index of the $j$ th coordinate process, $\left\{X_{n, j}: n \in \mathbb{Z}\right\}$, is equal to $\theta_{j}=\sum_{l \geq 1} \max _{k \in \mathbb{Z}} a_{l, k, j}$.

By (2.4), the expected number of exceedances over a high threshold in a block with at least one exceedance converges to the reciprocal of the extremal index. For univariate stationary processes, this characterization is due to Leadbetter (1983).

Finally, (2.5) admits two interpretations. The case $s_{n} / n \rightarrow 0$ states that the probability that the exceedance $X_{1} \not \leq n x$ is followed by a run of $s_{n}$ non-exceedances converges to $\theta(x)$, a property originally discovered by O'Brien (1987). The case $s_{n} / n \rightarrow \lambda>0$ can be reformulated as follows, with $T_{x}=\min \left\{i \geq 1: X_{i+1} \not \leq x\right\}$ :

$$
\lim _{n \rightarrow \infty} \operatorname{Pr}\left(\left\{V_{1}(x) / n\right\} T_{n x} \geq \lambda \mid X_{1} \not \leq n x\right)=\theta(x) \exp \{-\lambda \theta(x)\}, \quad \lambda>0 .
$$

In words, the normalized interarrival time $\left\{V_{1}(x) / n\right\} T_{n x}$ converges to the mixture distribution $\{1-\theta(x)\} \varepsilon_{0}+\theta(x) \operatorname{Exp}(\theta(x))$, where $\varepsilon_{0}$ is a point mass at 0 and $\operatorname{Exp}(\nu)$ is an exponential distribution with mean $1 / \nu$. For univariate sequences, a similar property was exploited by Ferro and Segers (2003) to construct an estimator for the extremal index; see also Chapter 10 of Beirlant et al. (2004).

\section{Variables in general state space}

\subsection{Setting}

Let $\left\{X_{n}: n \geq 1\right\}$ be a stationary sequence of random elements of a measurable space $(S, \delta)$, and let $B \in \delta$. Think of the random elements $X_{n}$ as representing the evolution of some system or process over time, and of the set $B$ as a failure set for which the events $\left\{X_{i} \in B\right\}$ have small probability but large repercussions if they occur. The archetypal situation is the one where the state space $S$ is the real line and the failure set $B$ is the open half-line $(u, \infty)$, the event $\left\{X_{i} \in B\right\}$ corresponding to the threshold exceedance $\left\{X_{i}>u\right\}$. In the example of M4 processes in Section 2, the state space is $\mathbb{R}^{d}$ and the failure set is of the form $\left\{y \in \mathbb{R}^{d}: y \not \leq x\right\}$. 
For $B \in \&$ and an integer $m \geq 1$, consider the following probabilities related to the occurrence of the events $\left\{X_{i} \in B\right\}$ :

$$
\begin{aligned}
p(B) & =\operatorname{Pr}\left(X_{1} \in B\right), \\
p_{m}(B) & =\operatorname{Pr}\left(\exists i=1, \ldots, m: X_{i} \in B\right), \\
q_{m}(B) & =1-p_{m}(B)=\operatorname{Pr}\left(X_{i} \notin B \forall i=1, \ldots, m\right) .
\end{aligned}
$$

To avoid trivialities, assume that $0<p(B)<1$. We will be interested in the asymptotics arising from a sequence of failure sets $B_{n} \in \delta$ such that the probability of a hit tends to 0 , i.e. $p\left(B_{n}\right) \rightarrow 0$.

\subsection{Quantities of interest}

From the above probabilities we can derive a number of quantities, all of which describe in a different way the dependence between failure set hits $\left\{X_{i} \in B\right\}$. If these events are independent, then simply $q_{m}(B)=\left\{q_{1}(B)\right\}^{m}$. In general, however, $q_{m}(B)=\left\{q_{1}(B)\right\}^{m \theta}$ for some $\theta=\theta_{m}^{\mathrm{M}}(B) \geq 0$, or, explicitly,

$$
\theta_{m}^{\mathrm{M}}(B)=\frac{\log q_{m}(B)}{m \log q_{1}(B)} .
$$

If $p_{m}(B)$ is small then $\theta_{m}^{\mathrm{M}}(B)$ is approximately equal to

$$
\theta_{m}^{\mathrm{B}}(B)=\frac{p_{m}(B)}{m p(B)} .
$$

Note that $\theta_{m}^{\mathrm{B}}(B)$ is equal to the reciprocal of the expected number of hits in the block $X_{1}, \ldots, X_{m}$, given that there is at least one hit, i.e.

$$
\mathrm{E}\left[\sum_{i=1}^{m} \mathbf{1}\left(X_{i} \in B\right) \mid \bigcup_{i=1}^{m}\left\{X_{i} \in B\right\}\right]=\frac{m p(B)}{p_{m}(B)}=\frac{1}{\theta_{m}^{\mathrm{B}}} .
$$

The conditional probability that a hit $\left\{X_{1} \in B\right\}$ is followed by a run of nonhits is

$$
\theta_{m}^{\mathrm{R}}(B)=\operatorname{Pr}\left(X_{i} \notin B \forall i=2, \ldots, m \mid X_{1} \in B\right)=\frac{p_{m}(B)-p_{m-1}(B)}{p(B)} .
$$

Conditionally on the process starting with a hit $\left\{X_{1} \in B\right\}$, the waiting time until the next hit is

$$
T_{B}=\min \left\{i \geq 1: X_{i+1} \in B\right\} .
$$

Its distribution is determined by

$$
\operatorname{Pr}\left(T_{B} \geq m \mid X_{1} \in B\right)=\theta_{m}^{\mathrm{R}}(B) .
$$

\subsection{Long-range dependence}

As our notation suggests, the quantities above turn out to be related, provided that the amount of long-range dependence is not too great. To control this, we impose conditions on a kind of mixing coefficient measuring the force of dependence, in a sample of size $n$, between blocks of variables of size at least $l$ that are separated by a gap of precisely $s$ :

$$
\alpha_{n, s, l}(B)=\max _{u, v, w}\left|\operatorname{Pr}\left(\bigcap_{u<i \leq v}\left\{X_{i} \notin B\right\} \cap \bigcap_{v<j \leq w}\left\{X_{j+s} \notin B\right\}\right)-q_{v-u}(B) q_{w-v}(B)\right| .
$$


Here the maximum ranges over all integers $u, v$, and $w$ such that $u \geq 0, v \geq u+l, w \geq v+l$, and $w+s \leq n$, and $l$ and $s$ are positive integers such that $2 l+s \leq n$. We write $\alpha_{n, l}(B)=\alpha_{n, l, l}(B)$ and $\bar{\alpha}_{n, l}(B)=\sup \left\{\alpha_{n, s, l}(B): l \leq s \leq n-2 l\right\}$, and for a real number $x$ denote by $\lfloor x\rfloor$ the largest integer not larger than $x$ and by $\lceil x\rceil$ the smallest integer not smaller than $x$.

\subsection{Characterization theorem}

Let $B_{n} \in \&$ be such that $0<p\left(B_{n}\right)<1$. Theorem 3.1 states the relations between the quantities $\theta_{m}^{\mathrm{M}}\left(B_{n}\right), \theta_{m}^{\mathrm{B}}\left(B_{n}\right)$, and $\theta_{m}^{\mathrm{R}}\left(B_{n}\right)$. It is an immediate corollary to the theorems in Section 5 applied to the events $A_{i, n}=\left\{X_{i} \in B_{n}\right\}$.

Theorem 3.1. Assume that there exists an integer sequence $l_{n}, 1 \leq l_{n} \leq n$, such that $l_{n}=o(n)$ and $\alpha_{n, l_{n}}\left(B_{n}\right) \rightarrow 0$.

(i) If $m_{n}, l_{n} \leq m_{n} \leq n$, is an integer sequence such that $l_{n}=o\left(m_{n}\right)$ and $\alpha_{n, l_{n}}=o\left[\max \left(m_{n} / n\right.\right.$, $\left.\left.p_{m_{n}}\left(B_{n}\right)\right)\right]$, then

$$
q_{n}\left(B_{n}\right)=\left\{q_{m_{n}}\left(B_{n}\right)\right\}^{n / m_{n}}+o(1) .
$$

In particular, $\lim \inf q_{n}\left(B_{n}\right) \geq \exp \left\{-\lim \sup n p\left(B_{n}\right)\right\}$.

(ii) If, additionally, $0<\lim \inf n p\left(B_{n}\right) \leq \lim \sup n p\left(B_{n}\right)<\infty$, then $\lim \sup \theta_{n}^{\mathrm{M}}\left(B_{n}\right) \leq 1$ and

$$
\lim _{n \rightarrow \infty} \sup _{m_{n} \leq i \leq j \leq n}\left|\theta_{i}^{\mathrm{M}}\left(B_{n}\right)-\theta_{j}^{\mathrm{M}}\left(B_{n}\right)\right|=0 .
$$

(iii) If, additionally, $m_{n}=o(n)$ then

$$
\theta_{n}^{\mathrm{M}}\left(B_{n}\right)=\theta_{m_{n}}^{\mathrm{B}}\left(B_{n}\right)+o(1)=\theta_{m_{n}}^{\mathrm{R}}\left(B_{n}\right)+o(1) .
$$

(iv) If, additionally, $\bar{\alpha}_{\lambda n, l_{n}}\left(B_{n}\right)=o(1)$ for every $\lambda>0$, then, for any sequence $\theta_{n}$ such that $\theta_{n}=\theta_{n}^{\mathrm{M}}\left(B_{n}\right)+o(1)$,

$$
\theta_{\left\lceil x / p\left(B_{n}\right)\right\rceil}^{\mathrm{R}}\left(B_{n}\right)=\operatorname{Pr}\left(p\left(B_{n}\right) T_{B_{n}} \geq x \mid X_{1} \in B_{n}\right)=\theta_{n} \exp \left\{-x \theta_{n}\right\}+o(1)
$$

locally uniformly in $x, 0<x<\infty$.

Remark 3.1. The condition that the process $\left\{X_{n}\right\}$ be stationary can be slightly weakened. It is sufficient that, for all positive integers $m$ and $n$, the probabilities $\operatorname{Pr}\left(X_{i+j} \in B_{n} \forall i=1, \ldots, m\right)$ do not depend on $j$; see also Definition 4.1 below.

Example 3.1. Without additional assumptions, M4 processes (2.1) satisfy a kind of mixing condition for rare events that makes Theorem 3.1 applicable to many failure sets other than those of the form $\left\{y \in \mathbb{R}^{d}: y \not \leq x\right\}$. For $x \in \mathbb{R}^{d}$ and $\lambda \in \mathbb{R}$, let (in obvious notation) $\max (x, \lambda)=\left(\max \left(x_{1}, \lambda\right), \ldots, \max \left(x_{d}, \lambda\right)\right)$. For $-\infty<r \leq s<\infty$ and $\lambda \in \mathbb{R}$, let $\sigma(r, s ; \lambda)$ be the $\sigma$-field generated by the random vectors $\left\{\max \left(X_{i}, \lambda\right): i \in \mathbb{Z} \cap[r, s]\right\}$. In this notation, every M4 process satisfies

$$
\max _{s=1, \ldots, v n-l_{n}} \sup _{\substack{A \in \sigma(1, s ; n \varepsilon) \\ B \in \sigma\left(s+l_{n}, v n ; n \varepsilon\right)}}|\operatorname{Pr}(A \cap B)-\operatorname{Pr}(A) \operatorname{Pr}(B)| \rightarrow 0
$$

for every $v, 0<v<\infty$, every positive integer sequence $l_{n}=1, \ldots, v n$ tending to infinity, and every $\varepsilon, 0<\varepsilon<\infty$. The proof of (3.1) is given in Appendix B. It is even possible to replace $\varepsilon$ by a positive sequence, $\varepsilon_{n}$, that tends to 0 sufficiently slowly. 
Note that for a finite set, $I$, of integers and for $u \in \mathbb{R}^{d}$, the event $\bigcap_{i \in I}\left\{X_{i} \leq u\right\}$ is contained in the $\sigma$-field $\sigma(r, s ; \lambda)$ if $I \subset \mathbb{Z} \cap[r, s]$ and $u_{j} \geq \lambda$ for every $j=1, \ldots, d$. In particular, by (3.1) all M4 processes satisfy the multivariate version of Leadbetter's $D\left(u_{n}\right)$ condition for every multivariate threshold sequence $u_{n}$ such that $\lim \inf u_{n, j} / n>0$ for every $j=1, \ldots, d$.

\section{Rare events: assumptions and notation}

Theorem 3.1 can be formulated completely in terms of the events $A_{i, n}=\left\{X_{i} \in B_{n}\right\}$; no reference needs to be made to the state space, the failure sets, or the random process. All we need is a triangular array, $\left\{A_{i, n}: n \geq 1,1 \leq i \leq n\right\}$, of events together with a notion of stationarity and restrictions on the amount of long-range dependence. The principal aim of this paper is to develop a theory of temporal dependence between rare events on this abstract level. In this section, we gather the ingredients that will appear in such a theory. The main results are stated in Section 5.

\subsection{Block stationarity}

Throughout, we will work with the following notion of stationarity for a vector of events $A_{1}, \ldots, A_{r}$.

Definition 4.1. Events $A_{1}, \ldots, A_{r}$ on a common probability space are called block stationary if $\operatorname{Pr}\left(\bigcup_{i=1}^{m} A_{i+j}\right)=\operatorname{Pr}\left(\bigcup_{i=1}^{m} A_{i}\right)$ for $m=1, \ldots, r-1$ and $j=1, \ldots, r-m$.

The probability that at least one of $m$ consecutive events occurs is

$$
p_{m}=\operatorname{Pr}\left(\bigcup_{i=1}^{m} A_{i+j}\right), \quad m=1, \ldots, r, j=0, \ldots, r-m .
$$

Thus, the probability that none of $m$ consecutive events occurs is

$$
q_{m}=1-p_{m}=\operatorname{Pr}\left(\bigcap_{i=1}^{m} A_{i+j}^{\mathrm{c}}\right), \quad m=1, \ldots, r, j=0, \ldots, r-m .
$$

For simplicity, we write $p=p_{1}$. To avoid trivialities, we henceforth assume that $0<p<1$. For positive integers $i$ and $j$ with $i+j \leq r$, we have

$$
p_{i} \leq p_{i+j} \leq p_{i}+p_{j} \quad \text { and } \quad q_{i+j} \leq q_{i} \leq q_{i+j}+p_{j} .
$$

Remark 4.1. If $r \geq 4$ then the property that events $A_{1}, \ldots, A_{r}$ are block stationary does not imply that the vector of indicator variables $\mathbf{1}\left(A_{1}\right), \ldots, \mathbf{1}\left(A_{r}\right)$ is stationary. (See Examples 4.1 and 4.2 for some counterexamples.) For the special case with three events $(r=3)$, block stationarity is the same as stationarity of the indicator variables, as kindly pointed out by a referee.

Example 4.1. Consider the discrete probability space $\Omega=\{1,2, \ldots, 16\}$ with uniform probabilities, and let

$$
\begin{aligned}
& A_{1}=\{1, \ldots, 8\}, \\
& A_{2}=\{1, \ldots, 4\} \cup\{9, \ldots, 12\}, \\
& A_{3}=\{1,2\} \cup\{5,6\} \cup\{9,10\} \cup\{13,14\}, \\
& A_{4}=\{1,2\} \cup\{9,10\} \cup\{3,7,11,15\} .
\end{aligned}
$$


Then $\operatorname{Pr}\left(A_{i}\right)=\frac{1}{2}$ for $i=1, \ldots, 4, \operatorname{Pr}\left(A_{i} \cup A_{i+1}\right)=\frac{3}{4}$ for $i=1,2,3$ and, for $i=1,2$, $\operatorname{Pr}\left(A_{i} \cup A_{i+1} \cup A_{i+2}\right)=\frac{7}{8}$. Hence, the events $A_{1}, \ldots, A_{4}$ are block stationary. However, $\operatorname{Pr}\left(A_{1} \cup A_{3}\right)=\frac{3}{4}$ while $\operatorname{Pr}\left(A_{2} \cup A_{4}\right)=\frac{5}{8}$, so the vector of corresponding indicator variables is not stationary.

Example 4.2. Let $Y_{n}$, where $n \in \mathbb{Z}$, be independent standard Fréchet random variables, i.e. $\operatorname{Pr}\left(Y_{n} \leq y\right)=\exp \{-1 / y\}$ for $0<y<\infty$. Furthermore, let $a_{i}, i \geq 0$, be nonnegative numbers such that $a_{i} \geq a_{i+1}$ for $i \geq 0$ and $\sum_{i \geq 0} a_{i}=1$. For positive integer $n$, let $\xi_{n}=\max \left\{a_{i} Y_{n-i}: i \geq 0\right\}$. The moving maximum process $\left\{\xi_{n}\right\}$ is stationary and $\operatorname{Pr}\left(\max _{i=1, \ldots, n} \xi_{i} \leq x\right)=\exp \left\{-\left\{(n-1) a_{0}+1\right\} / x\right\}$ for $0<x<\infty$.

Now let $\left\{\xi_{n}^{\prime}\right\}$ be another such moving maximum process, independent of $\left\{\xi_{n}\right\}$ and with parameters $a_{i}^{\prime}, i \geq 0$, where again $a_{i}^{\prime} \geq a_{i+1}^{\prime} \geq 0$ for $i \geq 0$ and $\sum_{i \geq 0} a_{i}^{\prime}=1$. Define a new process by intercalating $\left\{\xi_{n}\right\}$ and $\left\{\xi_{n}^{\prime}\right\}$ as $\left(X_{1}, X_{2}, X_{3}, X_{4}, \ldots\right)=\left(\xi_{1}, \xi_{1}^{\prime}, \xi_{2}, \xi_{2}^{\prime}, \ldots\right)$. If $a_{0}=a_{0}^{\prime}$ but $a_{i} \neq a_{i}^{\prime}$ for some $i \geq 1$, then the process $\left\{X_{n}\right\}$ is nonstationary. Nevertheless, the distribution of $\max \left\{X_{i+j}: i=1, \ldots, m\right\}$ does not depend on $j$ : for each real $x$, the events $A_{i}=\left\{X_{i}>x\right\}$ are block stationary.

\subsection{Quantities of interest}

Let $A_{1}, \ldots, A_{r}$ be a row of block stationary events. Recall $p_{m}$ and $q_{m}$ from (4.1) and (4.2). If the events are independent, then simply $q_{m}=q_{1}^{m}$ for all integer $m, 1 \leq m \leq r$. In general, however, $q_{m}=q_{1}^{m \theta}$ for some $\theta=\theta_{m}^{\mathrm{M}} \geq 0$, or, explicitly,

$$
\theta_{m}^{\mathrm{M}}=\frac{\log \left(q_{m}\right)}{m \log \left(q_{1}\right)}, \quad m=1, \ldots, r .
$$

If $p_{m}$ is small then $-\log \left(q_{m}\right)$ and $-\log \left(q_{1}\right)$ are approximately equal to $p_{m}$ and $p$, respectively. Substituting these approximations into (4.3) yields

$$
\theta_{m}^{\mathrm{B}}=\frac{p_{m}}{m p}, \quad m=1, \ldots, r .
$$

Note that $0<\theta_{m}^{\mathrm{B}} \leq 1$. The interpretation is that $1 / \theta_{m}^{\mathrm{B}}=m p / p_{m}$ is equal to the expected number of events that occur in a block of size $m$, given that there occurs at least one, i.e. $\mathrm{E}\left[\sum_{i=1}^{m} \mathbf{1}\left(A_{i}\right) \mid \bigcup_{i=1}^{m} A_{i}\right]=1 / \theta_{m}^{\mathrm{B}}$.

Conditionally on an event occurring, the probability that it is followed by a run of nonoccurring events is

$$
\theta_{m}^{\mathrm{R}}=\operatorname{Pr}\left(\bigcap_{i=2}^{m} A_{i}^{\mathrm{c}} \mid A_{1}\right)=\frac{p_{m}-p_{m-1}}{p}, \quad m=1, \ldots, r,
$$

where $p_{0}:=0$. By symmetry, $\theta_{m}^{\mathrm{R}}$ is also equal to the probability that an extreme event is not preceded by another one for a certain time, i.e. $\theta_{m}^{\mathrm{R}}=\operatorname{Pr}\left(\bigcap_{i=1}^{m-1} A_{i}^{\mathrm{c}} \mid A_{m}\right)$.

Finally, if the first event actually occurs, written $\omega \in A_{1}$, then the time to wait until the next event occurs is

$$
T(\omega)=\min \left\{j \geq 1: \omega \in A_{j+1}\right\} .
$$

(The minimum of the empty set is set to infinity, by convention.) The distribution of the interarrival time $T$ can be expressed as

$$
\operatorname{Pr}\left(T \geq t \mid A_{1}\right)=\theta_{t}^{\mathrm{R}}, \quad t=1, \ldots, r .
$$


The quantities $\theta_{m}^{\mathrm{M}}, \theta_{m}^{\mathrm{B}}$, and $\theta_{m}^{\mathrm{R}}$ are ordered in the following way.

Lemma 4.1. For integer $m, 1 \leq m \leq r$, we have $\theta_{m}^{\mathrm{R}} \leq \theta_{m}^{\mathrm{B}} \leq \theta_{m}^{\mathrm{M}} \leq \theta_{m}^{\mathrm{B}} / q_{m}$.

Proof. Since $\theta_{i}^{\mathrm{R}}$ is decreasing in $i$, we have

$$
p_{m}=\sum_{i=1}^{m}\left(p_{i}-p_{i-1}\right)=\sum_{i=1}^{m} p \theta_{i}^{\mathrm{R}} \geq m p \theta_{m}^{\mathrm{R}}
$$

whence $\theta_{m}^{\mathrm{R}} \leq \theta_{m}^{\mathrm{B}}$.

Next, the function $x \mapsto-x^{-1} \log (1-x)=\int_{0}^{1}(1-x y)^{-1} \mathrm{~d} y$ is increasing in $x<1$. Since $p_{m} \geq p$, we have $-p_{m}^{-1} \log \left(q_{m}\right) \geq-p^{-1} \log \left(q_{1}\right)$ and, thus, $\log \left(q_{m}\right) / \log \left(q_{1}\right) \geq p_{m} / p$, whence $\theta_{m}^{\mathrm{M}} \geq \theta_{m}^{\mathrm{B}}$.

Finally, as $x \leq-\log (1-x) \leq x /(1-x)$ for $0 \leq x<1$, we have $-\log \left(q_{m}\right) \leq p_{m} / q_{m}$ and $-\log \left(q_{1}\right) \geq p$, whence $\theta_{m}^{\mathrm{M}} \leq\left(p_{m} / q_{m}\right) /(m p)=\theta_{m}^{\mathrm{B}} / q_{m}$, completing the proof.

\subsection{Weak long-range dependence}

The amount of long-range dependence will be controlled by putting bounds on the coefficients

$$
\alpha_{s, l}:=\max _{v=l, \ldots, r-s-l} \max _{\substack{u=0, \ldots, v-l \\ w=v+l, \ldots, r-s}}\left|\operatorname{Pr}\left(\bigcap_{u<i \leq v} A_{i}^{\mathrm{c}} \cap \bigcap_{v<j \leq w} A_{j+s}^{\mathrm{c}}\right)-q_{v-u} q_{w-v}\right|,
$$

for $s=0, \ldots, r-2$ and $l=1, \ldots,\lfloor(r-s) / 2\rfloor$. The coefficient $\alpha_{s, l}$ describes the force of dependence between two blocks of length at least $l$ that are separated by a gap of size precisely $s$. We write $\alpha_{l}=\alpha_{l, l}$ and $\bar{\alpha}_{l}=\max \left\{\alpha_{s, l}: s=l, \ldots, r-2 l\right\}$.

The coefficients $\alpha_{s, l}$ were introduced by O'Brien (1987) in the classical setting of threshold exceedances $A_{i, n}=\left\{X_{i}>u_{n}\right\}$ in a stationary sequence $\left\{X_{n}\right\}$. More commonly used in this situation is Leadbetter's (1974) $D\left(u_{n}\right)$ condition, which, in our notation, is based on the coefficients

$$
\alpha_{s}^{D}:=\max _{j=1, \ldots, r-s-1} \max _{I, J}\left|\operatorname{Pr}\left(\bigcap_{i \in I \cup J} A_{i}^{\mathrm{c}}\right)-\operatorname{Pr}\left(\bigcap_{i \in I} A_{i}^{\mathrm{c}}\right) \operatorname{Pr}\left(\bigcap_{i \in J} A_{i}^{\mathrm{c}}\right)\right|, \quad s=0, \ldots, r-2,
$$

the maximum being over all non-empty subsets $I \subset\{1, \ldots, j\}$ and $J \subset\{j+s+1, \ldots, r\}$. Clearly $\max \left\{\alpha_{t, l}: t=s, \ldots, r-2 l\right\} \leq \alpha_{s}^{D}$ for $s=0, \ldots, r-2$, meaning that dependence restrictions based on the $\alpha_{s, l}$ are milder than the corresponding ones based on the $\alpha_{s}^{D}$. This improvement is useful, for example, for certain periodic Markov chains (O'Brien (1987)).

Observe that $\alpha_{s}^{D}$ is in turn smaller than

$$
\alpha_{s}^{\Delta}:=\max _{j=1, \ldots, r-s-1} \max _{E, F}|\operatorname{Pr}(E \cap F)-\operatorname{Pr}(E) \operatorname{Pr}(F)|, \quad s=0, \ldots, r-2,
$$

the maximum being over all $E \in \sigma\left(A_{1}, \ldots, A_{j}\right)$ and $F \in \sigma\left(A_{j+s+1}, \ldots, A_{r}\right)$. Bounds on $\alpha_{s}^{\Delta}$ are typically needed to establish the convergence of empirical point processes of exceedances to a compound Poisson process (Hsing et al. (1988), Barbour et al. (2002), Novak (2002)). 


\subsection{Triangular array of rare events}

The set-up for asymptotic results will be a triangular array of $A_{i, n}$ events, $n=1,2, \ldots, i=$ $1, \ldots, r_{n}$, for which every row $A_{1, n}, \ldots, A_{r_{n}, n}$ consists of block stationary events on a common probability space (which may vary with $n$ ). The probabilities of interest are

$$
p_{m, n}=\operatorname{Pr}\left(\bigcup_{i=1}^{m} A_{i+j, n}\right), \quad m=1, \ldots, r_{n}, j=0, \ldots, r_{n}-m,
$$

together with $q_{m, n}=1-p_{m, n}$ and $p_{n}=p_{1, n}$. The mixing coefficient (4.5) for the $n$th row is $\alpha_{s, l, n}$, and we write $\alpha_{l, n}=\alpha_{l, l, n}$ and $\bar{\alpha}_{l, n}=\max \left\{\alpha_{s, l, n}: s=l, \ldots, r_{n}-2 l\right\}$. Assume that $0<p_{n}<1$ for all $n$, and for $m=1, \ldots, r_{n}$ let

$$
\theta_{m, n}^{\mathrm{M}}=\frac{\log \left(q_{m, n}\right)}{m \log \left(q_{1, n}\right)}, \quad \theta_{m, n}^{\mathrm{B}}=\frac{p_{m, n}}{m p_{n}}, \quad \text { and } \quad \theta_{m, n}^{\mathrm{R}}=\frac{p_{m, n}-p_{m-1, n}}{p_{n}},
$$

where $p_{0, n}:=0$. The distribution of the interarrival time between the first event and the next one is

$$
\operatorname{Pr}\left(T_{n} \geq t \mid A_{1, n}\right)=\theta_{t, n}^{\mathrm{R}}, \quad t=1, \ldots, r_{n} .
$$

Finally, all asymptotic statements are to be understood as to hold $n \rightarrow \infty$.

\section{Main results}

The case of M4 processes in Section 2 suggests that properties of the extremal index of a univariate stationary sequence carry over to more general contexts. In this section, proper reformulations will be shown to remain true in the general setting of a triangular array $A_{1, n}, \ldots, A_{r_{n}, n}, n \geq 1$, of row-wise block stationary events of the type discussed in Section 4.4. The proofs of the results of this section depend on the results of Section 6 and are deferred to Appendix A.

\subsection{Big and small blocks}

For independent and identically distributed random variables $\left\{X_{n}\right\}$, the distribution of the sample maximum $M_{n}=\max \left(X_{1}, \ldots, X_{n}\right)$ is given by $\operatorname{Pr}\left(M_{n} \leq x\right)=\left\{\operatorname{Pr}\left(X_{1} \leq x\right)\right\}^{n}$. If the sequence is stationary, certain mixing conditions still guarantee that $\operatorname{Pr}\left(M_{r} \leq x\right)$ is close to $\left\{\operatorname{Pr}\left(M_{s} \leq x\right)\right\}^{r / s}$, provided that $r$ and $s$ are large enough. As a consequence, for such sequences the only nondegenerate weak limits of affinely normalized sample maxima are the extreme value distributions (Leadbetter (1974)). The argument can be extended to the multivariate case (Hsing (1989), Hüsler (1990)). Thus, in the general setting a natural question to ask is how closely the probability, $q_{r_{n}, n}$, of there being no extreme event in a row is approximated by the probability, $q_{s_{n}, n}^{r_{n} / s_{n}}$, of there being no extreme event in $r_{n} / s_{n}$ independent smaller blocks of size $s_{n}$.

Theorem 5.1. Assume that there exists an integer sequence $l_{n}, 1 \leq l_{n} \leq r_{n}$, such that $l_{n}=$ $o\left(r_{n}\right)$ and $\alpha_{l_{n}, n}=o(1)$. For every integer sequence $s_{n}, l_{n} \leq s_{n} \leq r_{n}$, such that $l_{n}=o\left(s_{n}\right)$ and $\alpha_{l_{n}, n}=o\left\{\max \left(s_{n} / r_{n}, p_{s_{n}, n}\right)\right\}$, we have

$$
q_{r_{n}, n}=q_{s_{n}, n}^{r_{n} / s_{n}}+o(1)
$$

Theorem 5.1 applies to any integer sequence $s_{n}$ with $l_{n} \leq s_{n} \leq r_{n}$ and $\lim \inf s_{n} / r_{n}>0$, and even to some with $s_{n}=o\left(r_{n}\right)$, for instance where $s_{n}$ is the integer part of $\max \left(\left(l_{n} r_{n}\right)^{1 / 2}, \alpha_{l_{n}, n}^{1 / 2} r_{n}\right)$. 


\subsection{Extremal index}

For univariate stationary sequences, the extremal index, whenever it exists, is defined through the relation $\operatorname{Pr}\left(M_{n} \leq u_{n}\right)=\left\{\operatorname{Pr}\left(X_{1} \leq u_{n}\right)\right\}^{n \theta}+o(1)$ for threshold sequences $u_{n}$ such that $0<\liminf n \operatorname{Pr}\left(X_{1}>u_{n}\right) \leq \lim \sup n \operatorname{Pr}\left(X_{1}>u_{n}\right)<\infty$. The extremal index typically arises as the reciprocal of the limit of the expected number of exceedances in a cluster (Leadbetter (1983)), and also as the limit probability that an exceedance is followed by a run of non-exceedances (O'Brien (1987)). These characterizations carry over to the general set-up of a triangular array of rare events. Recall the quantities $\theta_{m, n}^{\mathrm{M}}, \theta_{m, n}^{\mathrm{B}}$, and $\theta_{m, n}^{\mathrm{R}}$ from (4.6).

Theorem 5.2. Assume that there exists an integer sequence $l_{n}, 1 \leq l_{n} \leq r_{n}$, such that $l_{n}=$ $o\left(r_{n}\right)$ and $\alpha_{l_{n}, n}=o(1)$.

(i) If $\tau=\lim \sup r_{n} p_{n}<\infty$ then $\lim \inf q_{r_{n}, n} \geq \exp \{-\tau\}$ and $\lim \sup \theta_{r_{n}, n}^{\mathrm{M}} \leq 1$.

(ii) If, moreover, $\lim \inf r_{n} p_{n}>0$ then for every integer sequence $m_{n}, l_{n} \leq m_{n} \leq r_{n}$, such that $l_{n}=o\left(m_{n}\right)$ and $\alpha_{l_{n}, n}=o\left(m_{n} / r_{n}\right)$, we have

$$
\lim _{n \rightarrow \infty} \sup _{m_{n} \leq i \leq j \leq r_{n}}\left|\theta_{i, n}^{\mathrm{M}}-\theta_{j, n}^{\mathrm{M}}\right|=0 .
$$

If $p_{m_{n}, n}=o(1)$ then $\theta_{m_{n}, n}^{\mathrm{M}} \sim \theta_{m_{n}, n}^{\mathrm{B}}$, by Lemma 4.1. The following theorem relates $\theta_{m_{n}, n}^{\mathrm{M}}$ and $\theta_{m_{n}, n}^{\mathrm{B}}$ to $\theta_{m_{n}, n}^{\mathrm{R}}$.

Theorem 5.3. Assume that there exists an integer sequence $l_{n}, 1 \leq l_{n} \leq r_{n}$, such that $l_{n}=$ $o\left(r_{n}\right)$ and $\alpha_{l_{n}, n}=o(1)$.

(i) For every integer sequence $m_{n}, l_{n} \leq m_{n} \leq\left(r_{n}-l_{n}\right) / 2$, such that $l_{n}=o\left(m_{n}\right), p_{m_{n}, n}=o(1)$, and $\alpha_{l_{n}, n}=o\left(m_{n} p_{n}\right)$, we have $\theta_{m_{n}, n}^{\mathrm{R}}=\theta_{m_{n}, n}^{\mathrm{B}}+o(1)=\theta_{m_{n}, n}^{\mathrm{M}}+o(1)$.

(ii) If, moreover, $\alpha_{l_{n}, n}=o\left(p_{m_{n}, n}\right)$ then $\theta_{m_{n}, n}^{\mathrm{R}} \sim \theta_{m_{n}, n}^{\mathrm{B}} \sim \theta_{m_{n}, n}^{\mathrm{M}}$.

By definition, $q_{r_{n}, n}=q_{1, n}^{r_{n} \theta_{n}}$ with $\theta_{n}=\theta_{r_{n}, n}^{\mathrm{M}}$. The following theorem states conditions guaranteeing $q_{r_{n}, n}=q_{1, n}^{r_{n} \theta_{n}}+o(1)$ to hold for other choices of $\theta_{n}$.

Theorem 5.4. Assume that there exists an integer sequence $l_{n}, 1 \leq l_{n} \leq r_{n}$, such that $l_{n}=$ $o\left(r_{n}\right)$ and $\alpha_{l_{n}, n}=o(1)$.

(i) For every integer sequence $m_{n}, l_{n} \leq m_{n} \leq r_{n}$, such that $l_{n}=o\left(m_{n}\right), m_{n}=o\left(r_{n}\right)$, and $\alpha_{l_{n}, n}=o\left\{\max \left(m_{n} / r_{n}, p_{m_{n}, n}\right)\right\}$, we have

$$
q_{r_{n}, n}=q_{1, n}^{r_{n} \theta_{n}}+o(1)=\exp \left(-r_{n} p_{n} \theta_{n}\right)+o(1)
$$

for $\theta_{n} \in\left\{\theta_{m_{n}, n}^{\mathrm{M}}, \theta_{m_{n}, n}^{\mathrm{B}}\right\}$.

(ii) If, moreover, $p_{m_{n}, n} \rightarrow 0$ then (5.1) remains true with $\theta_{n}=\theta_{m_{n}, n}^{\mathrm{R}}$.

Remark 5.1. Without the extra condition $p_{m_{n}, n} \rightarrow 0$, part (ii) of Theorem 5.4 is not true. Consider, for example, independent events with $p_{n} \rightarrow 0, r_{n} \sim p_{n}^{-3}$, and $m_{n} \sim p_{n}^{-2}$ : on the one hand $q_{r_{n}, n}=\left(1-p_{n}\right)^{r_{n}} \rightarrow 0$, while on the other hand

$$
r_{n} \theta_{m_{n}, n}^{\mathrm{R}}=p_{n}^{-3}\left(1-p_{n}\right)^{m_{n}-1}=p_{n}^{-3} \exp \left\{-p_{n}^{-1}(1+o(1))\right\} \rightarrow 0 .
$$

The condition $p_{m_{n}, n} \rightarrow 0$ is implied by each one of the following: (i) $m_{n} p_{n} \rightarrow 0$, (ii) $\lim \sup _{n \rightarrow \infty} r_{n} p_{n}<\infty$, (iii) $\liminf _{n \rightarrow \infty} q_{r_{n}, n}>0$. Regarding (i), just observe that 
$p_{m_{n}, n} \leq m_{n} p_{n}$. Since $m_{n}=o\left(r_{n}\right)$, (ii) implies (i), and since $q_{r_{n}, n}=\left(1-p_{m_{n}, n}\right)^{r_{n} / m_{n}}+o(1)$, by Theorem 5.1, condition (iii) is also sufficient.

\subsection{Interarrival times}

Next we focus on the interarrival time, $T_{n}$, between the occurrence of the event $A_{1, n}$ and the occurrence of the first subsequent event, conditionally on $A_{1, n}$ (see (4.7)). Since the probability of a single event is $p_{n}$, the average interarrival time should be $1 / p_{n}$, regardless of the dependence structure. Under certain conditions, the standardized interarrival time $p_{n} T_{n}$ converges weakly to a nondegenerate limit. Recall that

$$
\bar{\alpha}_{l, n}=\max \left\{\alpha_{s, l, n}: s=l, \ldots, r_{n}-2 l\right\},
$$

with $\alpha_{s, l, n}$ as in (4.5) for the row $A_{1, n}, \ldots, A_{r_{n}, n}$.

Theorem 5.5. If $0<\lim \inf r_{n} p_{n} \leq \lim \sup r_{n} p_{n}<\infty$ and there exists an integer sequence $l_{n}, 1 \leq l_{n} \leq r_{n}$, such that $l_{n}=o\left(r_{n}\right)$ and $\bar{\alpha}_{l_{n}, n}=o(1)$, then, for every sequence $\theta_{n}$ such that $\theta_{n}=\theta_{r_{n}, n}^{\mathrm{M}}+o(1)$

$$
\operatorname{Pr}\left(p_{n} T_{n} \geq x \mid A_{1, n}\right)=\theta_{n} \exp \left\{-x \theta_{n}\right\}+o(1)
$$

locally uniformly in $x, 0<x<\lim \inf r_{n} p_{n}$.

By (5.1), the normalized interarrival time $p_{n} T_{n}$ is approximately distributed according to the mixture distribution $\left(1-\theta_{n}\right) \varepsilon_{0}+\theta_{n} \operatorname{Exp}\left(\theta_{n}\right)$. The point mass at $0, \varepsilon_{0}$, describes the interarrival times between events within a cluster, while the exponential part describes the interarrival times between different clusters. This interpretation is in accordance with the compound Poisson limit (established under stronger mixing conditions) for the empirical point process of occurrence times of exceedances over a high threshold in a univariate stationary sequence (Hsing et al. (1988)). It was exploited by Ferro and Segers (2003) in the construction of an estimator for the extremal index.

\section{Finite-sample inequalities}

The key to the asymptotic results of Section 5 is a collection of sharp inequalities that apply in the setting of a single row, $A_{1}, \ldots, A_{r}$, of block stationary events. Throughout this section, we employ the notation of Sections 4.1, 4.2, and 4.3.

\subsection{Big and small blocks}

The first lemma exploits an idea of Loynes (1965): a large block can be broken into approximately independent smaller blocks by removing an asymptotically negligible number of events from between the smaller blocks and using the appropriate mixing coefficients. By convention, the sum over the empty set is equal to 0 and the product over the empty set is equal to 1 .

Lemma 6.1. For integer $a_{1}, b_{1}, \ldots, a_{k}, b_{k} \in\{0, \ldots, r\}$ such that there exists a positive integer $l$ satisfying $b_{i}-a_{i} \geq l$ for all $i=1, \ldots, k$ and $a_{i+1}-b_{i}=l$ for all $i=1, \ldots, k-1$, we have

$$
-\left(\alpha_{l}+p_{l}\right) \sum_{i=2}^{k} \prod_{j=i+1}^{k} q_{b_{j}-a_{j}} \leq q_{b_{k}-a_{1}}-\prod_{i=1}^{k} q_{b_{i}-a_{i}} \leq \alpha_{l} \sum_{i=2}^{k} \prod_{j=i+1}^{k} q_{b_{j}-a_{j}} .
$$


Proof. We proceed by induction on $k$. For $k=1$, there is nothing to prove, so consider $k \geq 2$. We have

$$
q_{b_{k}-a_{1}}=\operatorname{Pr}\left(\bigcap_{i=a_{1}+1}^{b_{k}} A_{i}^{\mathrm{c}}\right)\left\{\begin{array}{l}
\leq \operatorname{Pr}\left(\bigcap_{i=a_{1}+1}^{b_{k-1}} A_{i}^{\mathrm{c}} \cap \bigcap_{i=a_{k}+1}^{b_{k}} A_{i}^{\mathrm{c}}\right), \\
\geq \operatorname{Pr}\left(\bigcap_{i=a_{1}+1}^{b_{k-1}} A_{i}^{\mathrm{c}} \cap \bigcap_{i=a_{k}+1}^{b_{k}} A_{i}^{\mathrm{c}}\right)-\operatorname{Pr}\left(\bigcup_{i=b_{k-1}+1}^{a_{k}} A_{i}\right) .
\end{array}\right.
$$

Moreover,

$$
\left|\operatorname{Pr}\left(\bigcap_{i=a_{1}+1}^{b_{k-1}} A_{i}^{\mathrm{c}} \cap \bigcap_{i=a_{k}+1}^{b_{k}} A_{i}^{\mathrm{c}}\right)-q_{b_{k-1}-a_{1}} q_{b_{k}-a_{k}}\right| \leq \alpha_{l} .
$$

Combining these, we find that

$$
q_{b_{k-1}-a_{1}} q_{b_{k}-a_{k}}-\alpha_{l}-p_{l} \leq q_{b_{k}-a_{1}} \leq q_{b_{k-1}-a_{1}} q_{b_{k}-a_{k}}+\alpha_{l} .
$$

Applying the induction hypothesis on $q_{b_{k-1}-a_{1}}$ completes the proof.

A useful special case of Lemma 6.1 is when the sizes, $b_{i}-a_{i}$, of the smaller blocks are all the same.

Lemma 6.2. For integer $l$ and $m, 1 \leq l \leq m \leq r$, and $k, 1 \leq k \leq\lfloor(r+l) /(m+l)\rfloor$, we have

$$
q_{r} \leq q_{m}^{k}+\frac{\alpha_{l}}{\max \left(m / r, p_{m}\right)} .
$$

If, additionally, $2 l+m \leq r$ then, for $k=\lceil(r+l) /(m+l)\rceil$,

$$
q_{r} \geq q_{m}^{k}-\frac{\alpha_{l}+p_{l}}{\max \left(m / r, p_{m}\right)} .
$$

Proof. Let $k=1, \ldots,\lfloor(r+l) /(m+l)\rfloor$ and set $a_{i}=(i-1)(m+l)$ and $b_{i}=a_{i}+m$ for $i=1, \ldots, k$. The integers $a_{1}, b_{1}, \ldots, a_{k}, b_{k}$ satisfy the conditions of Lemma 6.1; in particular, $b_{k}=k m+(k-1) l \leq r$. Hence,

$$
-\left(\alpha_{l}+p_{l}\right) \sum_{i=2}^{k} q_{m}^{k-i} \leq q_{k m+(k-1) l}-q_{m}^{k} \leq \alpha_{l} \sum_{i=2}^{k} q_{m}^{k-i} .
$$

Now, $\sum_{i=2}^{k} q_{m}^{k-i}=\left(1-q_{m}^{k-1}\right) /\left(1-q_{m}\right)$ and, furthermore, for $x, 0<x<1$, and $a \geq$ 1 or $a=0$, we have $1-x^{a} \leq \min (a(1-x)$, 1) by the mean value theorem, and thus $\left(1-x^{a}\right) /(1-x) \leq \min \{a, 1 /(1-x)\}$. Hence, for $k=1, \ldots,\lfloor(r+l) /(m+l)\rfloor$,

$$
-\left(\alpha_{l}+p_{l}\right) \min \left(k-1,1 / p_{m}\right) \leq q_{k m+(k-1) l}-q_{m}^{k} \leq \alpha_{l} \min \left(k-1,1 / p_{m}\right) .
$$

Since $q_{r} \leq q_{k m+(k-1) l}$, we obtain (6.1).

Next, suppose that $2 l+m \leq r$. Applying Lemma 6.1 with $a_{1}=0, b_{1}=m, a_{2}=m+l$, and $b_{2}=r$ yields

$$
q_{r} \geq q_{m} q_{r-m-l}-\left(\alpha_{l}+p_{l}\right)
$$


Let $k=\lceil(r+l) /(m+l)\rceil$. Since $r-m-l \leq(k-1)(m+l)-l \leq r$, by the first inequality of (6.3), we have

$$
q_{r-m-l} \geq q_{(k-1)(m+l)-l} \geq q_{m}^{k-1}-\left(\alpha_{l}+p_{l}\right) \min \left(k-2,1 / p_{m}\right) .
$$

Combining the previous two displays gives

$$
q_{r} \geq q_{m}^{k}-\left(\alpha_{l}+p_{l}\right)\left\{q_{m} \min \left(k-2,1 / p_{m}\right)+1\right\} \geq q_{m}^{k}-\left(\alpha_{l}+p_{l}\right) \min \left(k-1,1 / p_{m}\right),
$$

from which we obtain (6.2). This completes the proof of the lemma.

Lemma 6.2 leads to inequalities involving $q_{r}-q_{m}^{r / m}$ when $m$ is small compared to $r$.

Lemma 6.3. For positive integer $m, l \leq m \leq r$, we have

$$
q_{r} \leq q_{m}^{r / m}+\frac{\alpha_{l}}{\max \left(m / r, p_{m}\right)}+\frac{l}{m}+\frac{m}{r} .
$$

If, additionally, $2 l+m \leq r$ then

$$
q_{r} \geq q_{m}^{r / m}-\frac{\alpha_{l}+p_{l}}{\max \left(m / r, p_{m}\right)}-\frac{l}{m}-\frac{m}{r} .
$$

Proof. By the mean value theorem,

$$
\left|x^{a}-x^{b}\right| \leq \max (1-a / b, 1-b / a), \quad 0 \leq x \leq 1, a>0, b>0 .
$$

Let $k=\lfloor(r+l) /(m+l)\rfloor$. Since $(r-m) /(m+l) \leq k \leq r / m$, we have

$$
q_{m}^{k}-q_{m}^{r / m} \leq 1-m k / r \leq l / m+m / r
$$

Combining this with (6.1) yields (6.4).

Next, suppose that $2 l+m \leq r$. Let $k=\lceil(r+l) /(m+l)\rceil$. By (6.6), we have

$$
\left|q_{m}^{k}-q_{m}^{r / m}\right| \leq \max (1-m k / r, 1-r /(m k))
$$

Since $r /(m+l) \leq k<r / m+1$, we have $1-m k / r \leq l / m$ and $1-r /(m k) \leq m / r$, whence $\max (1-m k / r, 1-r /(m k)) \leq l / m+m / r$. Combining this with (6.2) yields (6.5). This completes the proof of the lemma.

\subsection{The extremal index}

The quantities $\theta_{m}^{\mathrm{M}}=\log \left(q_{m}\right) /\left\{m \log \left(q_{1}\right)\right\}$ of (4.3) are approximately constant over a wide range of $m$.

Lemma 6.4. For integer $l$ and $m, 1 \leq l \leq m \leq r$, such that $2 l+m \leq r$, and with $\tau=r p$ and $\varepsilon=(r / m) \alpha_{l}+(1+\tau) l / m+m / r$, we have

$$
\left|\theta_{r}^{\mathrm{M}}-\theta_{m}^{\mathrm{M}}\right| \leq \frac{\varepsilon}{\tau(\exp \{-\tau\}-(\tau / 2)(m / r)-\varepsilon)_{+}} .
$$


Proof. By Lemma 6.3, $\left|q_{r}-q_{m}^{r / m}\right| \leq \varepsilon$. Also, $q_{r}=\exp \left\{r \log \left(q_{1}\right) \theta_{r}^{\mathrm{M}}\right\}$ and $q_{m}^{r / m}=$ $\exp \left\{r \log \left(q_{1}\right) \theta_{m}^{\mathrm{M}}\right\}$ and, by the mean value theorem,

$$
r\left|\log \left(q_{1}\right)\right|\left|\theta_{r}^{\mathrm{M}}-\theta_{m}^{\mathrm{M}}\right| \min \left(q_{r}, q_{m}^{r / m}\right) \leq \varepsilon .
$$

Since $q_{r} \geq q_{m}^{r / m}-\varepsilon$ and $\left|\log \left(q_{1}\right)\right| \geq p$, we have

$$
\left|\theta_{r}^{\mathrm{M}}-\theta_{m}^{\mathrm{M}}\right| \leq \frac{\varepsilon}{\tau\left(q_{m}^{r / m}-\varepsilon\right)_{+}} .
$$

As $\exp \{-a x\}-(1-x)^{a} \leq x / 2$ for $0 \leq x \leq 1$ and $a \geq 1$, we have $q_{m}^{r / m}=\left(1-p_{m}\right)^{r / m} \geq$ $\exp \left\{-(r / m) p_{m}\right\}-p_{m} / 2$. Applying the inequality $p_{m} \leq m p$ completes the proof.

In Lemma 4.1 we have already seen that $\theta_{m}^{\mathrm{R}} \leq \theta_{m}^{\mathrm{B}}$. Here is a converse inequality.

Lemma 6.5. For integer $l$ and $m, 1 \leq l \leq m \leq r$, such that $2 m+l \leq r$, we have

$$
\theta_{m}^{\mathrm{R}} \geq \theta_{m}^{\mathrm{B}}-\frac{p_{m}^{2}}{m p}-\frac{\alpha_{l}+p_{l}}{m p} .
$$

Proof. We have

$$
p_{m}=\operatorname{Pr}\left(\bigcup_{i=1}^{m} A_{i} \cap \bigcap_{i=m+1}^{2 m+l} A_{i}^{\mathrm{c}}\right)+\operatorname{Pr}\left(\bigcup_{i=1}^{m} A_{i} \cap \bigcup_{i=m+1}^{2 m+l} A_{i}\right) .
$$

On the one hand

$$
\operatorname{Pr}\left(\bigcup_{i=1}^{m} A_{i} \cap \bigcap_{i=m+1}^{2 m+l} A_{i}^{\mathrm{c}}\right)=\sum_{i=1}^{m} \operatorname{Pr}\left(A_{i} \cap \bigcap_{j=i+1}^{2 m+l} A_{j}^{\mathrm{c}}\right)=\sum_{i=1}^{m} p \theta_{2 m+l-i+1}^{\mathrm{R}} \leq m p \theta_{m}^{\mathrm{R}},
$$

while on the other hand

$$
\operatorname{Pr}\left(\bigcup_{i=1}^{m} A_{i} \cap \bigcup_{i=m+1}^{2 m+l} A_{i}\right) \leq \operatorname{Pr}\left(\bigcup_{i=1}^{m} A_{i} \cap \bigcup_{i=m+l+1}^{2 m+l} A_{i}\right)+p_{l} \leq p_{m}^{2}+\alpha_{l}+p_{l} .
$$

Combining the previous three displays yields

$$
m p \theta_{m}^{\mathrm{R}} \geq p_{m}-p_{m}^{2}-\alpha_{l}-p_{l},
$$

and dividing by $m p$ yields (6.7). This completes the proof of the lemma.

By definition, $q_{r}=q_{1}^{r \theta}$ with $\theta=\theta_{r}^{\mathrm{M}}$. The following lemma gives bounds on the error induced by choosing $\theta$ to equal $\theta_{m}^{\mathrm{B}}$ or $\theta_{m}^{\mathrm{R}}$. Note that $q_{1}^{r \theta} \leq \exp \{-r p \theta\}$ for $\theta \geq 0$.

Lemma 6.6. For integer $l$ and $m, 1 \leq l \leq m \leq r$, and $\theta_{m} \in\left\{\theta_{m}^{\mathrm{B}}, \theta_{m}^{\mathrm{R}}\right\}$, we have

$$
q_{r} \leq q_{1}^{r \theta_{m}}+\frac{\alpha_{l}}{\max \left(m / r, p_{m}\right)}+\frac{l}{m}+\frac{m}{r} .
$$

If, additionally, $2 l+m \leq r$ then

$$
q_{r} \geq \exp \left\{-r p \theta_{m}^{\mathrm{B}}\right\}-\frac{\alpha_{l}+p_{l}}{\max \left(m / r, p_{m}\right)}-\frac{l}{m}-2 \frac{m}{r} .
$$


If, additionally, $2 m+l \leq r$ then

$$
q_{r} \geq \exp \left\{-r p \theta_{m}^{\mathrm{R}}\right\}-3 \frac{\alpha_{l}+p_{l}}{\max \left(m / r, p_{m}\right)}-\frac{l}{m}-2 \frac{m}{r}-2 p_{m} .
$$

Proof. Note that $1-a x \leq(1-x)^{a}$ for $0 \leq x \leq 1$ and $a \geq 1$. As $m \theta_{m}^{\mathrm{B}}=p_{m} / p \geq 1$, we have

$$
q_{m}^{r / m}=\left(1-p_{m}\right)^{r / m}=\left(1-m \theta_{m}^{\mathrm{B}} p\right)^{r / m} \leq(1-p)^{r \theta_{m}^{\mathrm{B}}} .
$$

Since $\theta_{m}^{\mathrm{B}} \geq \theta_{m}^{\mathrm{R}}$, by Lemma 4.1, we also have $q_{m}^{r / m} \leq(1-p)^{r \theta_{m}^{\mathrm{R}}}$. In combination with (6.4), this yields (6.8).

To prove (6.9) we start from (6.5). We need to find suitable lower bounds for $q_{m}^{r / m}$. For $0 \leq$ $x \leq 1$ and $a \geq 1$, we have

$$
\begin{aligned}
0 & \leq \exp \{-a x\}-(1-x)^{a} \\
& \leq(\exp \{-x\}-(1-x)) a \exp \{-(a-1) x\} \\
& \leq \frac{x^{2}}{2} a \exp \{1-a x\} \\
& =\frac{1}{a} \frac{\exp \{1\}}{2}(a x)^{2} \exp \{-a x\} \\
& \leq \frac{1}{a}
\end{aligned}
$$

since $\sup _{y \geq 0} y^{2} \exp \{-y\}=4 \exp \{-2\}$. Hence,

$$
q_{m}^{r / m}=\left(1-p_{m}\right)^{r / m} \geq \exp \left\{-(r / m) p_{m}\right\}-\frac{m}{r}=\exp \left\{-r p \theta_{m}^{\mathrm{B}}\right\}-\frac{m}{r},
$$

which, in combination with (6.5), yields (6.9).

Finally, we will apply Lemma 6.5 to the difference between $\theta_{m}^{\mathrm{B}}$ and $\theta_{m}^{\mathrm{R}}$, to convert the lower bound for $q_{r}$ in terms of $\theta_{m}^{\mathrm{B}}$ into a lower bound in terms of $\theta_{m}^{\mathrm{R}}$. Since $\exp \{z\}=\exp \{z / 2\}^{2} \geq$ $(1+z / 2)^{2}$ for $z \geq 0$, for $0 \leq x \leq y$ we have

$$
\begin{aligned}
0 & \leq \exp \{-x\}-\exp (-y) \\
& =\int_{x}^{y} \exp \{-z\} \mathrm{d} z \\
& \leq \int_{x}^{y}(1+z / 2)^{-2} \mathrm{~d} z \\
& \leq \frac{y-x}{1+y / 2}
\end{aligned}
$$

Hence, by (6.7) we have

$$
\begin{aligned}
\exp \left\{-r p \theta_{m}^{\mathrm{R}}\right\}-\exp \left\{-r p \theta_{m}^{\mathrm{B}}\right\} & \leq \frac{r p\left(\theta_{m}^{\mathrm{B}}-\theta_{m}^{\mathrm{R}}\right)}{1+r p \theta_{m}^{\mathrm{B}} / 2} \\
& \leq \frac{r p\left\{p_{m}^{2} /(m p)+\left(\alpha_{l}+p_{l}\right) /(m p)\right\}}{1+(r / m) p_{m} / 2} \\
& =\frac{(r / m) p_{m}^{2}+\left(\alpha_{l}+p_{l}\right) /(m / r)}{1+(r / m) p_{m} / 2}
\end{aligned}
$$


If, on the one hand, $m / r \geq p_{m}$ then

$$
\exp \left\{-r p \theta_{m}^{\mathrm{R}}\right\}-\exp \left\{-r p \theta_{m}^{\mathrm{B}}\right\} \leq p_{m}+\frac{\alpha_{l}+p_{l}}{m / r},
$$

while if, on the other hand, $m / r<p_{m}$ then

$$
\exp \left\{-r p \theta_{m}^{\mathrm{R}}\right\}-\exp \left\{-r p \theta_{m}^{\mathrm{B}}\right\} \leq 2\left(p_{m}+\frac{\alpha_{l}+p_{l}}{p_{m}}\right) .
$$

Thus,

$$
\exp \left\{-r p \theta_{m}^{\mathrm{R}}\right\}-\exp \left\{-r p \theta_{m}^{\mathrm{B}}\right\} \leq 2\left(p_{m}+\frac{\alpha_{l}+p_{l}}{\max \left(m / r, p_{m}\right)}\right) .
$$

Combining this with $(6.11)$ yields

$$
q_{m}^{r / m} \geq \exp \left\{-r p \theta_{m}^{\mathrm{R}}\right\}-\frac{m}{r}-2 p_{m}-2 \frac{\alpha_{l}+p_{l}}{\max \left(m / r, p_{m}\right)} .
$$

In turn, this inequality, in combination with (6.5), yields (6.10). The proof of the lemma is thus complete.

\subsection{Interarrival times}

Conditionally on $A_{1}$, the distribution of the time, $T$, until the next event is

$$
\operatorname{Pr}\left(T \geq s \mid A_{1}\right)=\operatorname{Pr}\left(\bigcap_{i=2}^{s} A_{i}^{\mathrm{c}} \mid A_{1}\right)=\theta_{s}^{\mathrm{R}}, \quad s=1, \ldots, r
$$

(see (4.4)). We break up the block $\bigcap_{i=2}^{s} A_{i}^{\mathrm{c}}$ into an initial smaller block, $\bigcap_{i=2}^{m} A_{i}^{\mathrm{c}}$, and a subsequent larger block, $\bigcap_{i=m+1}^{s} A_{i}^{\mathrm{c}}$. The next lemma demonstrates how to control the dependence between $A_{1}$ and the initial block, on the one hand, and the subsequent block, on the other. Recall that $\bar{\alpha}_{l}=\max \left\{\alpha_{s, l}: s=l, \ldots, r-2 l\right\}$, with $\alpha_{s, l}$ as in (4.5).

Lemma 6.7. For integer $l$ and $m, 1 \leq l \leq m \leq r$, such that $2 m+l \leq r$, and for integer $s$, $m+l \leq s \leq r-m$, we have

$$
-\frac{\alpha_{l}+p_{l}}{m p} \leq \theta_{s}^{\mathrm{R}}-\theta_{m}^{\mathrm{B}} q_{s} \leq 2 \frac{\bar{\alpha}_{l}}{m p}+p_{m}+p_{l} .
$$

Proof. For integer $t, m+1 \leq t \leq r$, we have

$$
\operatorname{Pr}\left(\bigcup_{i=1}^{m} A_{i} \cap \bigcap_{i=m+1}^{t} A_{i}^{\mathrm{c}}\right)=\sum_{k=1}^{m} \operatorname{Pr}\left(A_{k} \cap \bigcap_{i=k+1}^{t} A_{i}^{\mathrm{c}}\right)=\sum_{k=1}^{m} p \theta_{t-k+1}^{\mathrm{R}},
$$

implying that

$$
m p \theta_{t}^{\mathrm{R}} \leq \operatorname{Pr}\left(\bigcup_{i=1}^{m} A_{i} \cap \bigcap_{i=m+1}^{t} A_{i}^{\mathrm{c}}\right) \leq m p \theta_{t-m}^{\mathrm{R}} .
$$

Hence, for integer $s, m+1 \leq s \leq r-m$, we have

$$
\operatorname{Pr}\left(\bigcup_{i=1}^{m} A_{i} \cap \bigcap_{i=m+1}^{s+m} A_{i}^{\mathrm{c}}\right) \leq m p \theta_{s}^{\mathrm{R}} \leq \operatorname{Pr}\left(\bigcup_{i=1}^{m} A_{i} \cap \bigcap_{i=m+1}^{s} A_{i}^{\mathrm{c}}\right) .
$$


Now,

$$
\begin{aligned}
0 & \leq \operatorname{Pr}\left(\bigcup_{i=1}^{m} A_{i} \cap \bigcap_{i=m+1}^{s} A_{i}^{\mathrm{c}}\right)-\operatorname{Pr}\left(\bigcup_{i=1}^{m} A_{i} \cap \bigcap_{i=m+1}^{s+m} A_{i}^{\mathrm{c}}\right) \\
& \leq \operatorname{Pr}\left(\bigcup_{i=1}^{m} A_{i} \cap \bigcup_{i=s+1}^{s+m} A_{i}\right) \\
& \leq p_{m}^{2}+\alpha_{s-m, l} .
\end{aligned}
$$

Moreover,

$$
\begin{aligned}
0 & \leq \operatorname{Pr}\left(\bigcup_{i=1}^{m} A_{i} \cap \bigcap_{i=m+l+1}^{s+m} A_{i}^{\mathrm{c}}\right)-\operatorname{Pr}\left(\bigcup_{i=1}^{m} A_{i} \cap \bigcap_{i=m+1}^{s+m} A_{i}^{\mathrm{c}}\right) \\
& \leq \operatorname{Pr}\left(\bigcup_{i=m+1}^{m+l} A_{i}\right) \\
& =p_{l}
\end{aligned}
$$

and, if $s \geq m+l$,

$$
\left|\operatorname{Pr}\left(\bigcup_{i=1}^{m} A_{i} \cap \bigcap_{i=m+l+1}^{s+m} A_{i}^{\mathrm{c}}\right)-p_{m} q_{s-l}\right| \leq \alpha_{l} .
$$

Combining the four previous displays yields

$$
p_{m} q_{s-l}-\alpha_{l}-p_{l} \leq m p \theta_{s}^{\mathrm{R}} \leq p_{m} q_{s-l}+\alpha_{l}+p_{m}^{2}+\alpha_{s-m, l},
$$

or, dividing by $m p$,

$$
\theta_{m}^{\mathrm{B}} q_{s-l}-\frac{\alpha_{l}+p_{l}}{m p} \leq \theta_{s}^{\mathrm{R}} \leq \theta_{m}^{\mathrm{B}} q_{s-l}+\frac{\alpha_{l}+\alpha_{s-m, l}}{m p}+p_{m},
$$

as $p_{m} \leq m p$. That $q_{s} \leq q_{s-l} \leq q_{s}+p_{l}$ then concludes the proof.

\section{Appendix A. Proofs for Section 5}

Recall the setting of a triangular array of events, as discussed in Section 4.4. The probability, $p_{l_{n}, n}$, of the blocks that are cut away is dealt with in the next lemma.

Lemma A.1. Let $l_{n}$ and $m_{n}, 1 \leq l_{n} \leq m_{n} \leq r_{n}$, be integers with $l_{n}=o\left(m_{n}\right)$.

(i) Let $0<\lambda_{n} \rightarrow 0$. If $p_{m_{n}, n}=O\left(\lambda_{n}\right)$ and $\alpha_{l_{n}, n}=o\left(\lambda_{n}\right)$, then $p_{l_{n}, n}=o\left(\lambda_{n}\right)$.

(ii) If $0<p_{m_{n}, n} \rightarrow 0$ and $\alpha_{l_{n}, n}=o\left(p_{m_{n}, n}\right)$, then $p_{l_{n}, n}=o\left(p_{m_{n}, n}\right)$.

Proof. (i) Let $k$ be a positive integer. If $n$ is large enough that $(2 k+1) l_{n} \leq m_{n}$, then by (6.1), with the choices $l=l_{n}, m=l_{n}$, and $r=(2 k+1) l_{n}$, we have

$$
\begin{aligned}
1-p_{m_{n}, n} & \leq 1-p_{(2 k+1) l_{n}, n} \\
& \leq\left(1-p_{l_{n}, n}\right)^{k}+(2 k+1) \alpha_{l_{n}, n} \\
& \leq \exp \left(-p_{l_{n}, n} k\right)+(2 k+1) \alpha_{l_{n}, n} .
\end{aligned}
$$


If $n$ is also large enough that $p_{m_{n}, n}+(2 k+1) \alpha_{l_{n}, n}<1$, then, as $-\log (1-x) \leq x /(1-x)$ for $x<1$, we have

$$
\begin{aligned}
p_{l_{n}, n} & \leq-\frac{1}{k} \log \left(1-p_{m_{n}, n}-(2 k+1) \alpha_{l_{n}, n}\right) \\
& \leq \frac{1}{k} \frac{p_{m_{n}, n}+(2 k+1) \alpha_{l_{n}, n}}{1-p_{m_{n}, n}-(2 k+1) \alpha_{l_{n}, n}} .
\end{aligned}
$$

Hence, $\lim \sup p_{l_{n}, n} / \lambda_{n} \leq k^{-1} \lim \sup p_{m_{n}, n} / \lambda_{n}$. Letting $k \rightarrow \infty$ shows that $p_{l_{n}, n} / \lambda_{n} \rightarrow 0$.

(ii) To prove this part, simply take $\lambda_{n}=p_{m_{n}, n}$ in part (i).

Proof of Theorem 5.1. Without loss of generality, we can restrict $n$ to a subsequence along which $s_{n} / r_{n}$ converges to some limit $\lambda \in[0,1]$.

Suppose first that $\lambda=0$. By the first inequality of Lemma 6.3, $q_{r_{n}, n} \leq q_{s_{n}, n}^{r_{n} / s_{n}}+o(1)$. Now consider a further subsequence along which $\mu_{n}:=\left(r_{n} / s_{n}\right) p_{s_{n}, n}$ converges to some limit $\mu \in[0, \infty]$. If $\mu=\infty$ then $q_{s_{n}, n}^{r_{n} / s_{n}}=\left\{1-\left(s_{n} / r_{n}\right) \mu_{n}\right\}^{r_{n} / s_{n}} \rightarrow 0$ and, hence, $q_{r_{n}, n} \rightarrow 0$ along this subsequence. If $\mu<\infty$ then, again along the second subsequence, $p_{s_{n}, n}=O\left(s_{n} / r_{n}\right)$ and, thus, by assumption, $\alpha_{l_{n}, n}=o\left(p_{s_{n}, n}\right)$. By the second inequality of Lemma 6.3 and by Lemma A.1(ii), it follows from this that also $q_{r_{n}, n} \geq q_{s_{n}, n}^{r_{n} / s_{n}}+o(1)$.

If instead $\lambda>0$ then choose a positive integer sequence $m_{n}, l_{n} \leq m_{n} \leq s_{n}$, such that $l_{n}=o\left(m_{n}\right), m_{n}=o\left(s_{n}\right)$, and $\alpha_{l_{n}, n}=o\left(m_{n} / s_{n}\right)$; for instance, take $m_{n}$ to equal the integer part of $\max \left(\left(l_{n} s_{n}\right)^{1 / 2}, \alpha_{l_{n}, n}^{1 / 2} s_{n}\right)$. From the arguments in the $\lambda=0$ case, we have $q_{r_{n}, n}=q_{m_{n}, n}^{r_{n} / m_{n}}+o(1)$ and $q_{s_{n}, n}=q_{m_{n}, n}^{s_{n} / m_{n}}+o(1)$. As $r_{n} / s_{n} \sim 1 / \lambda$, we also have

$$
q_{s_{n}, n}^{r_{n} / s_{n}}=q_{s_{n}, n}^{1 / \lambda}+o(1)=q_{m_{n}, n}^{\left(s_{n} / m_{n}\right)(1 / \lambda)}+o(1)=q_{m_{n}, n}^{r_{n} / m_{n}}+o(1) .
$$

Proof of Theorem 5.2. (i) Let $m_{n}, l_{n} \leq m_{n} \leq r_{n}$, be an integer sequence such that $l_{n}=$ $o\left(m_{n}\right), m_{n}=o\left(r_{n}\right)$, and $\alpha_{l_{n}, n}=o\left(m_{n} / r_{n}\right)$; for instance, let $m_{n}$ be the integer part of $\max \left(\left(l_{n} r_{n}\right)^{1 / 2}, \alpha_{l_{n}, n}^{1 / 2} r_{n}\right)$. By Theorem 5.1, $q_{r_{n}, n}=q_{m_{n}, n}^{r_{n} / m_{n}}+o(1)$. Since $r_{n} p_{n}=O(1)$ and $m_{n}=o\left(r_{n}\right)$, we have $p_{m_{n}, n} \leq m_{n} p_{n}=o(1)$. Hence,

$$
q_{m_{n}, n}^{r_{n} / m_{n}}=\exp \left\{-\left(r_{n} / m_{n}\right) p_{m_{n}, n}\right\}+o(1) \geq \exp \left\{-r_{n} p_{n}\right\}+o(1) .
$$

Without loss of generality, suppose that $r_{n} p_{n} \rightarrow \tau \in[0, \infty)$. If $\tau=0$ then $\theta_{r_{n}, n}^{\mathrm{M}} \leq$ $1 / q_{r_{n}, n} \rightarrow 1$, by Lemma 4.1. If $\tau>0$ then $\theta_{r_{n}, n}^{\mathrm{M}}=\theta_{m_{n}, n}^{\mathrm{M}}+o(1)$, by Lemma 6.4, and $\theta_{m_{n}, n}^{\mathrm{M}} \leq$ $1 / q_{m_{n}, n} \rightarrow 1$, by Lemma 4.1 .

(ii) Without loss of generality, suppose that $m_{n}=o\left(r_{n}\right)$; otherwise, apply a construction as in part (i). We have to show that $\theta_{i_{n}, n}^{\mathrm{M}}=\theta_{j_{n}, n}^{\mathrm{M}}+o(1)$ for all positive integer sequences $i_{n}$ and $j_{n}$ such that $m_{n} \leq i_{n} \leq j_{n} \leq r_{n}$. By restricting to a subsequence, if necessary, we can assume that $i_{n} / r_{n} \rightarrow \lambda$ and $j_{n} / r_{n} \rightarrow \mu$ for some $\lambda$ and $\mu, 0 \leq \lambda \leq \mu \leq 1$. If $\lambda=0$ or $\mu=0$, then by Lemma 6.4 we have $\theta_{i_{n}, n}^{\mathrm{M}}=\theta_{r_{n}, n}^{\mathrm{M}}+o(1)$. If instead $\lambda>0$ or $\mu>0$, then by Lemma 6.4 we have $\theta_{i_{n}, n}^{\mathrm{M}}=\theta_{m_{n}, n}^{\mathrm{M}}+o(1)$. Moreover, as $\theta_{m_{n}, n}^{\mathrm{M}}=\theta_{r_{n}, n}^{\mathrm{M}}+o(1)$, we obtain $\theta_{i_{n}, n}^{\mathrm{M}}=\theta_{j_{n}, n}^{\mathrm{M}}+o(1)$ in all cases. The proof of the theorem is thus complete.

Proof of Theorem 5.3. (i) From Lemmas 4.1 and 6.5, we have

$$
\theta_{m_{n}, n}^{\mathrm{B}}-\frac{p_{m_{n}, n}^{2}}{m_{n} p_{n}}-\frac{\alpha_{l_{n}, n}+p_{l_{n}, n}}{m_{n} p_{n}} \leq \theta_{m_{n}, n}^{\mathrm{R}} \leq \theta_{m_{n}, n}^{\mathrm{B}} .
$$

Since $p_{m_{n}, n} \leq m_{n} p_{n}$ and $p_{l_{n}, n} \leq l_{n} p_{n}$, the conditions imply that $\theta_{m_{n}, n}^{\mathrm{R}}=\theta_{m_{n}, n}^{\mathrm{B}}+o(1)$. 
(ii) From the above display, we have

$$
\theta_{m_{n}, n}^{\mathrm{B}}\left(1-p_{m_{n}, n}-\frac{\alpha_{l_{n}, n}+p_{l_{n}, n}}{p_{m_{n}, n}}\right) \leq \theta_{m_{n}, n}^{\mathrm{R}} \leq \theta_{m_{n}, n}^{\mathrm{B}} .
$$

As $p_{l_{n}, n}=o\left(p_{m_{n}, n}\right)$ by Lemma A.1(ii), in fact $\theta_{m_{n}, n}^{\mathrm{R}} \sim \theta_{m_{n}, n}^{\mathrm{B}}$. Moreover, by Lemma 4.1, $\theta_{m_{n}, n}^{\mathrm{B}} \leq \theta_{m_{n}, n}^{\mathrm{M}} \leq \theta_{m_{n}, n}^{\mathrm{B}} / q_{m_{n}, n} \sim \theta_{m_{n}, n}^{\mathrm{B}}$.

Proof of Theorem 5.4. From (6.8),

$$
q_{r_{n}, n} \leq q_{1, n}^{r_{n} \theta_{n}}+o(1) \leq \exp \left\{-r_{n} p_{n} \theta_{n}\right\}+o(1),
$$

for $\theta_{n} \in\left\{\theta_{m_{n}, n}^{\mathrm{B}}, \theta_{m_{n}, n}^{\mathrm{R}}\right\}$. Without loss of generality, fix a subsequence along which $p_{m_{n}, n}$ converges to some $p \in[0,1]$.

For $p>0$, since $\theta_{m_{n}, n}^{\mathrm{B}} \leq \theta_{m_{n}, n}^{\mathrm{M}}$ (see Lemma 4.1), we have

$$
\exp \left\{-r_{n} p_{n} \theta_{m_{n}, n}^{\mathrm{M}}\right\} \leq \exp \left\{-r_{n} p_{n} \theta_{m_{n}, n}^{\mathrm{B}}\right\}=\exp \left\{-\left(r_{n} / m_{n}\right) p_{m_{n}, n}\right\}=o(1),
$$

implying that $q_{r_{n}, n}, q_{1, n}^{r_{n} \theta_{n}}$, and $\exp \left\{-r_{n} p_{n} \theta_{n}\right\}$ are all $o(1)$ for $\theta_{n} \in\left\{\theta_{m_{n}, n}^{\mathrm{B}}, \theta_{m_{n}, n}^{\mathrm{M}}\right\}$.

For $p=0$, we have $p_{l_{n}, n}=o\left\{\max \left(m_{n} / r_{n}, p_{m_{n}, n}\right)\right\}$ by Lemma A.1(i); hence, from (6.9) and (6.10),

$$
q_{r_{n}, n} \geq \exp \left\{-r_{n} p_{n} \theta_{n}\right\}+o(1) \geq q_{1, n}^{r_{n} \theta_{n}}+o(1)
$$

for $\theta_{n} \in\left\{\theta_{m_{n}, n}^{\mathrm{B}}, \theta_{m_{n}, n}^{\mathrm{R}}\right\}$. In combination with the first display of this proof, this yields

$$
q_{r_{n}, n}=\exp \left\{-r_{n} p_{n} \theta_{n}\right\}+o(1) \geq q_{1, n}^{r_{n} \theta_{n}}+o(1)
$$

for $\theta_{n} \in\left\{\theta_{m_{n}, n}^{\mathrm{B}}, \theta_{m_{n}, n}^{\mathrm{R}}\right\}$. As $p_{m_{n}, n} \rightarrow 0$ implies that $\theta_{m_{n}, n}^{\mathrm{M}} \sim \theta_{m_{n}, n}^{\mathrm{B}}$, by Lemma 4.1, the above display remains valid for $\theta_{n}=\theta_{m_{n}, n}^{\mathrm{M}}$ because $a_{n}^{1+\varepsilon_{n}}=a_{n}+o(1)$ for any real sequences $a_{n}, 0 \leq a_{n} \leq 1$, and $\varepsilon_{n} \rightarrow 0$.

Proof of Theorem 5.5. Let $m_{n}, l_{n} \leq m_{n} \leq r_{n}$, be an integer sequence such that $l_{n}=o\left(m_{n}\right)$, $m_{n}=o\left(r_{n}\right)$, and $\bar{\alpha}_{l_{n}, n}=o\left(m_{n} / r_{n}\right)$. From Lemma 6.7,

$$
\max \left\{\left|\theta_{s, n}^{\mathrm{R}}-\theta_{m_{n}, n}^{\mathrm{B}} q_{s, n}\right|: s=m_{n}+l_{n}, \ldots, r_{n}-m_{n}\right\} \rightarrow 0 .
$$

Hence, for any integer sequence $s_{n}, m_{n}+l_{n} \leq s_{n} \leq r_{n}-m_{n}$, we have $\theta_{s_{n}, n}^{\mathrm{R}}=\theta_{m_{n}, n}^{\mathrm{B}} q_{s_{n}, n}+o(1)$. Also, by Theorem 5.4, $q_{s_{n}, n}=\exp \left\{-s_{n} p_{n} \theta_{m_{n}, n}^{\mathrm{B}}\right\}+o(1)$. For $0<x<\lim \inf r_{n} p_{n}$, the sequence $s_{n}=\left\lceil x / p_{n}\right\rceil$ falls in the required range, and we find that

$$
\theta_{\left\lceil x / p_{n}\right\rceil, n}^{\mathrm{R}}=\theta_{m_{n}, n}^{\mathrm{B}} \exp \left\{-x \theta_{m_{n}, n}^{\mathrm{B}}\right\}+o(1)
$$

locally uniformly in $x, 0<x<\liminf r_{n} p_{n}$.

Observe that $\theta_{m_{n}, n}^{\mathrm{B}}=\theta_{m_{n}, n}^{\mathrm{M}}+o(1)=\theta_{r_{n}, n}^{\mathrm{M}}+o(1)$ (see Theorems 5.3(i) and 5.2(ii)) and, for nonnegative $\theta, \theta^{\prime}$, and $x$, that $\left|\theta \exp \{-x \theta\}-\theta^{\prime} \exp \left\{-x \theta^{\prime}\right\}\right| \leq\left|\theta-\theta^{\prime}\right|$; this completes the proof. 


\section{Appendix B. Proof of (3.1)}

Fix a positive integer $m$. For $i \in \mathbb{Z}$ and $j=1, \ldots, d$, let

$$
\begin{aligned}
X_{i, j}^{(m)} & =\max _{l \geq 1} \max _{|k|<m} a_{l, k, j} Z_{l, i-k}, \\
R_{i, j}^{(m)} & =\max _{l \geq 1} \max _{|k| \geq m} a_{l, k, j} Z_{l, i-k} .
\end{aligned}
$$

Observe that $X_{i, j}=\max \left(X_{i, j}^{(m)}, R_{i, j}^{(m)}\right)$.

Let $b_{l, k}=\max _{j=1, \ldots, d} a_{l, k, j}$. For $0<\varepsilon<\infty$,

$$
\begin{aligned}
\operatorname{Pr} & \left(\exists i=1, \ldots, v n, j=1, \ldots, d: R_{i, j}^{(m)}>n \varepsilon\right) \\
& =\operatorname{Pr}\left(\exists i=1, \ldots, v n, j=1, \ldots, d, l \geq 1,|k| \geq m: a_{l, k, j} Z_{l, i-k}>n \varepsilon\right) \\
& =\operatorname{Pr}\left(\exists i=1, \ldots, v n, l \geq 1,|k| \geq m: b_{l, k} Z_{l, i-k}>n \varepsilon\right) \\
& =\operatorname{Pr}\left(\exists l \geq 1, p \in \mathbb{Z}: \max _{|k| \geq m, 1 \leq k+p \leq v n} b_{l, k} Z_{l, p}>n \varepsilon\right) \\
& \leq \frac{1}{n \varepsilon} \sum_{l \geq 1} \sum_{p \in \mathbb{Z}} \max _{|k| \geq m, 1 \leq k+p \leq v n} b_{l, k} .
\end{aligned}
$$

Replacing the last maximum by a summation and interchanging the summation over $p$ with the resulting summation over $k$ gives

$$
\operatorname{Pr}\left(\exists i=1, \ldots, \nu n, j=1, \ldots, d: R_{i, j}^{(m)}>n \varepsilon\right) \leq \frac{v}{\varepsilon} \sum_{l \geq 1} \sum_{|k| \geq m} b_{l, k} .
$$

Now let $X_{i}^{(m)}=\left(X_{i, 1}^{(m)}, \ldots, X_{i, d}^{(m)}\right)$. Then

$$
\begin{aligned}
& \operatorname{Pr}\left(\max \left(X_{i}^{(m)}, n \varepsilon\right)=\max \left(X_{i}, n \varepsilon\right) \forall i=1, \ldots, v n\right) \\
& \geq 1-\operatorname{Pr}\left(\exists i=1, \ldots, v n, j=1, \ldots, d: R_{i, j}^{(m)}>n \varepsilon\right),
\end{aligned}
$$

implying that, by (B.1),

$$
\operatorname{Pr}\left(\max \left(X_{i}^{\left(m_{n}\right)}, n \varepsilon\right)=\max \left(X_{i}, n \varepsilon\right) \forall i=1, \ldots, v n\right) \rightarrow 1
$$

for every positive integer sequence, $m_{n}$, tending to infinity. Equation (3.1) now follows from the fact that the process $\left\{X_{i}^{(m)}: i \in \mathbb{Z}\right\}$ is $2 m$-dependent in the sense that $\left\{X_{i}^{(m)}: i \leq r-m\right\}$ and $\left\{X_{i}^{(m)}: i \geq r+m\right\}$ are independent for every integer $r$.

\section{Acknowledgements}

Gratefully acknowledged are encouraging comments by John Einmahl and Jürg Hüsler, as well as a meticulous reading combined with useful and constructive suggestions by an anonymous referee. The author's research was supported by a postdoctoral grant from the Fund for Scientific Research, Flanders, and a VENI grant from the Dutch Science Foundation (NWO). 


\section{References}

Barbour, A. D., Novak, S. Y. and Xia, A. (2002). Compound Poisson approximation for the distribution of extremes. Adv. Appl. Prob. 24, 223-240.

Beirlant, J., Goegebeur, Y., Segers, J. and Teugels, J. (2004). Statistics of Extremes: Theory and Applications. John Wiley, New York.

De HaAn, L. (1984). A spectral representation for max-stable processes. Ann. Prob. 12, 1194-1204.

Ferro, C. A. T. and Segers, J. (2003). Inference for clusters of extremes. J. R. Statist. Soc. B 65, 545-556.

Hsing, T. (1989). Extreme value theory for multivariate stationary sequences. J. Multivariate Anal. 29, $274-291$.

Hsing, T., HüSlER, J. AND LeAdBetTer, M. R. (1988). On the exceedance point process for a stationary sequence. Prob. Theory Relat. Fields 78, 97-112.

HÜSLER, J. (1990). Multivariate extreme values in stationary random sequences. Stoch. Process. Appl. 35, 99-108.

Hüsler, J. (1993). A note on exceedances and rare events of non-stationary sequences. J. Appl. Prob. 30, 877-888.

Hüsler, J. AND Schmidt, M. (1996). A note on the point processes of rare events. J. Appl. Prob. 33, $654-663$.

LeAdbetter, M. R. (1974). On extreme values in stationary sequences. Z. Wahrscheinlichkeitsth. 28, $289-303$.

LEADBETTER, M. R. (1983). Extremes and local dependence of stationary sequences. Z. Wahrscheinlichkeitsth. 65, 291-306.

Loynes, R. M. (1965). Extreme values in uniformly mixing stationary stochastic processes. Ann. Math. Statist. 36, 993-999.

Nandagopalan, S. (1994). On the multivariate extremal index. J. Res. Nat. Inst. Stand. Technol. 99, 543-550.

Novak, S. Y. (2002). Multilevel clustering of extremes. Stoch. Process. Appl. 97, 59-75.

O’Brien, G. L. (1987). Extreme values for stationary and Markov sequences. Ann. Prob. 15, 281-291.

Perfekt, R. (1997). Extreme value theory for a class of Markov chains with values in $\mathbb{R}^{d}$. Adv. Appl. Prob. 29, 138-164.

Smith, R. L. AND Weissman, I. (1996). Characterization and estimation of the multivariate extremal index. Tech. Rep., University of North Carolina. Available at www.unc.edu/depts/statistics/postscript/rs/extremal.ps.

ZHANG, Z. (2002). Multivariate extremes, max-stable process estimation and dynamic financial modeling. Doctoral Thesis, University of North Carolina.

Zhang, Z. AND Smith, R. L. (2004). The behavior of multivariate maxima of moving maxima processes. J. Appl. Prob. 41, 1113-1123. 